\title{
Positive association of the oriented percolation cluster in randomly oriented graphs
}

\author{
François Bienvenu ${ }^{* \dagger}$
}

July 21, 2020

\begin{abstract}
Consider any fixed graph whose edges have been randomly and independently oriented, and write $\{S \rightsquigarrow i\}$ to indicate that there is an oriented path going from a vertex $s \in S$ to vertex $i$. Narayanan (2016) proved that for any set $S$ and any two vertices $i$ and $j,\{S \rightsquigarrow i\}$ and $\{S \rightsquigarrow j\}$ are positively correlated. His proof relies on the Ahlswede-Daykin inequality, a rather advanced tool of probabilistic combinatorics.

In this short note, I give an elementary proof of the following, stronger result: writing $V$ for the vertex set of the graph, for any source set $S$, the events $\{S \rightsquigarrow i\}, i \in V$, are positively associated - meaning that the expectation of the product of increasing functionals of the family $\{S \rightsquigarrow i\}$ for $i \in V$ is greater than the product of their expectations.
\end{abstract}

\section{Introduction}

Oriented percolation is the study of connectivity in a random oriented graph. In most settings, one starts from a graph with a fixed orientation and then keeps each edge with a given probability. Classical such models include the north-east lattice [3] and the hypercube [5].

Another broad and natural class of random oriented graphs is obtained by starting from a fixed graph and then orienting each edge, independently of the orientations of other edges. Note that, in the general case, the orientations of the edges need not be unbiased: some edges can be allowed to have a higher probability to point towards one of their ends than towards the other. Percolation on such randomly oriented graphs has been studied, e.g. in [7], and more recently in [8], which motivated the present work.

In [8], Narayanan showed that if the edges of any fixed graph are randomly and independently oriented, then writing $\{S \rightsquigarrow i\}$ to indicate that there is an oriented path going from a vertex $s \in S$ to vertex $i$, we have

$$
\mathbb{P}(S \rightsquigarrow i, S \rightsquigarrow j) \geqslant \mathbb{P}(S \rightsquigarrow i) \mathbb{P}(S \rightsquigarrow j) .
$$

The aim of this note is to strengthen and simplify the proof of this result. More specifically, let $V$ be the vertex set of the graph. We prove that the events $\{S \rightsquigarrow i\}$, $i \in V$, are positively associated, without resorting to advanced results such as the Ahlswede-Daykin inequality [1].

\footnotetext{
${ }^{*}$ Center for Interdisciplinary Research in Biology (CIRB), CNRS UMR 7241, Collège de France, Paris, France.

${ }^{\dagger}$ Laboratoire de Probabilités, Statistique et Modélisation (LPSM), CNRS UMR 8001, Sorbonne Université, Paris, France

E-mail: francois.bienvenu@normalesup.org
} 


\subsection{Positive association and related notions}

There are many ways to formalize the idea of a positive dependence between the random variables of a family $\mathbf{X}=\left(X_{i}\right)_{i \in I}$. A straightforward, weak one is to ask that these variables be pairwise positively correlated, i.e.

$$
\forall i, j \in I, \quad \mathbb{E}\left(X_{i} X_{j}\right) \geqslant \mathbb{E}\left(X_{i}\right) \mathbb{E}\left(X_{j}\right) .
$$

A much stronger condition, due to [4], is known as positive association. In the following definition and throughout the rest of this note, we use bold letters to denote vectors, as in $\mathbf{X}=\left(X_{i}\right)_{i \in I}$, and we write $\mathbf{X} \leqslant \mathbf{X}^{\prime}$ to say that $X_{i} \leqslant X_{i}^{\prime}$ for all $i$. Finally, a function $f: \mathbb{R}^{I} \rightarrow \mathbb{R}$ is said to be increasing when $\mathbf{X} \leqslant \mathbf{X}^{\prime} \Longrightarrow$ $f(\mathbf{X}) \leqslant f\left(\mathbf{X}^{\prime}\right)$.

Definition 1.1. The random vector $\mathbf{X}=\left(X_{i}\right)_{i \in I}$ is said to be positively associated when, for all increasing functions $f$ and $g$,

$$
\mathbb{E}(f(\mathbf{X}) g(\mathbf{X})) \geqslant \mathbb{E}(f(\mathbf{X})) \mathbb{E}(g(\mathbf{X}))
$$

whenever these expectations exist.

Without further mention, we only consider test functions $f$ and $g$ for which $\mathbb{E}(f(\mathbf{X})), \mathbb{E}(g(\mathbf{X}))$ and $\mathbb{E}(f(\mathbf{X}) g(\mathbf{X}))$ exist.

We say that the events $A_{i}, i \in I$, are positively associated when the corresponding vector of indicator variables $\left(\mathbb{1}_{A_{i}}\right)_{i \in I}$ is positively associated. Similarly, a random subset $R$ of the fixed set $I$ can be seen as the vector

$$
\mathbf{R}=\left(\mathbb{1}_{\{i \in R\}}\right)_{i \in I},
$$

so that $R$ is said to be positively associated when the events $\{i \in R\}, i \in I$, are. This is equivalent to saying that for any increasing functions $f$ and $g$ from the power set of $I$ to $\mathbb{R}$,

$$
\mathbb{E}(f(R) g(R)) \geqslant \mathbb{E}(f(R)) \mathbb{E}(g(R)),
$$

where $f$ being increasing is understood to mean that $r^{\prime} \subset r \Longrightarrow f\left(r^{\prime}\right) \leqslant f(r)$.

Positive association is famous for the FKG theorem, which states that it is implied by a lattice condition that can sometimes be very easy to check [6]. Another reason why it is so useful is that it implies weaker positive dependence notions that have to be checked in applications. One example of this is the existence of increasing couplings and the corresponding notion of positive relation used in the Stein-Chen method - see e.g, [2] and [9].

\subsection{Notation}

Let us fix some notation to be used throughout the rest of this document.

We study the simple graph $G=(V, E)$. Unless explicitly specified otherwise, $V$ is assumed to be finite and we denote by $|V|$ its cardinality. The edges of $G$ have a random orientation that is independent of the orientations of other edges and we write $\{i \rightarrow j\}$ to indicate that the edge $\{i j\}$ is oriented towards $j$. Formally, we are 
thus given a family of events $(\{i \rightarrow j\},\{i j\} \in E)$ such that $\{i \rightarrow j\}=\{j \rightarrow i\}^{\mathrm{c}}$ and for all $\{i j\},\{i \rightarrow j\} \Perp(\{k \rightarrow \ell\},\{k \ell\} \neq\{i j\})$.

Finally, for every pair of vertices $i$ and $j$, we write $\{i \rightsquigarrow j\}$ for the event that there exists an oriented path going from $i$ to $j$. Similarly, for every source set $S$ we let $\{S \rightsquigarrow i\}=\bigcup_{j \in S}\{j \rightsquigarrow i\}$ be the event that there is an oriented path from $S$ to $i$, and for every target set $T$ we let $\{i \rightsquigarrow T\}=\bigcup_{j \in T}\{i \rightsquigarrow j\}$ be the event that there is an oriented path from $i$ to $T$. If there is an ambiguity regarding which graph is considered for these events, we will specify it with the notation $\{i \stackrel{G}{\rightsquigarrow} j\}$.

\section{Positive association of the percolation cluster}

\subsection{Preliminary lemma}

Lemma 2.1. Let $\Gamma$ be a finite set and let $R$ be a positively associated random subset of $\Gamma$. Let $X_{i}^{r}, r \subset \Gamma$ and $i \in V$, be a family of events on the same probability space as $R$ with the property that

(i) $r^{\prime} \subset r \Longrightarrow X_{i}^{r^{\prime}} \subset X_{i}^{r}, \forall i \in V$.

(ii) For all $r \subset \Gamma,\left(X_{i}^{r}\right)_{i \in V}$ is positively associated and independent of $R$.

For all $i \in V$, define $X_{i}^{R}$ by

$$
X_{i}^{R}:=\bigcup_{r \subset \Gamma}\{R=r\} \cap X_{i}^{r} .
$$

Then, the events $X_{i}^{R}, i \in V$, are positively associated.

Proof. Let $f$ and $g$ be two increasing functions. We have

$$
\begin{aligned}
\mathbb{E}\left(f\left(\mathbf{X}^{R}\right) g\left(\mathbf{X}^{R}\right)\right) & =\sum_{r \subset \Gamma} \mathbb{E}\left(f\left(\mathbf{X}^{r}\right) g\left(\mathbf{X}^{r}\right) \mathbb{1}_{\{R=r\}}\right) \\
& =\sum_{r \subset \Gamma} \mathbb{E}\left(f\left(\mathbf{X}^{r}\right) g\left(\mathbf{X}^{r}\right)\right) \mathbb{P}(R=r) \\
& \geqslant \sum_{r \subset \Gamma} \mathbb{E}\left(f\left(\mathbf{X}^{r}\right)\right) \mathbb{E}\left(g\left(\mathbf{X}^{r}\right)\right) \mathbb{P}(R=r),
\end{aligned}
$$

because $\mathbf{X}^{r} \Perp R$ and $\mathbf{X}^{r}$ is positively associated. Now, let $u: r \mapsto \mathbb{E}\left(f\left(\mathbf{X}^{r}\right)\right)$ and $v: r \mapsto \mathbb{E}\left(g\left(\mathbf{X}^{r}\right)\right)$, so that the last sum is $\mathbb{E}(u(R) v(R))$. Note that $u$ and $v$ are increasing, since $f$ and $g$ are and, by hypothesis, $r^{\prime} \subset r \Longrightarrow \mathbf{X}^{r^{\prime}} \leqslant \mathbf{X}^{r}$. Therefore, by the positive association of $R$,

$$
\mathbb{E}(u(R) v(R)) \geqslant \mathbb{E}(u(R)) \mathbb{E}(v(R)) .
$$

Finally, using again the independence of $\mathbf{X}^{r}$ and $R$, we have $\mathbb{E}(u(R))=\mathbb{E}\left(f\left(\mathbf{X}^{R}\right)\right)$ and $\mathbb{E}(v(R))=\mathbb{E}\left(g\left(\mathbf{X}^{R}\right)\right)$, which concludes the proof. 


\subsection{Main result}

Theorem 2.2. Let $G$ be a finite graph with vertex set $V$, whose edges have been randomly and independently oriented. Then, for any source set $S$, the events $\{S \rightsquigarrow i\}, i \in V$, are positively associated, i.e., for all increasing functions $f$ and $g$ and writing $\mathbf{X}=\left(\mathbb{1}_{\{S \sim i\}}\right)_{i \in V}$,

$$
\mathbb{E}(f(\mathbf{X}) g(\mathbf{X})) \geqslant \mathbb{E}(f(\mathbf{X})) \mathbb{E}(g(\mathbf{X}))
$$

Proof. Our proof uses the same induction on the number of vertices as Narayanan's. The difference is that we use Lemma 2.1 rather than the Ahlswede-Daykin inequality to propagate the positive dependence.

The theorem is trivial for the graph consisting of a single vertex (a family of a single variable being always positively associated) so let us assume that it holds for every graph with strictly less than $|V|$ vertices.

Let $\Gamma$ be the neighborhood of $S$, i.e.

$$
\Gamma=\{v \in V \backslash S: \exists s \in S \text { s.t. }\{v s\} \in E\} .
$$

Then, let $R$ be the random subset of $\Gamma$ defined by

$$
R=\{v \in \Gamma: \exists s \in S \text { s.t. } s \rightarrow v\} .
$$

Observe that the events $\{i \in R\}, i \in \Gamma$ are independent, so that the set $R$ is positively associated.

Next, let $H$ be the subgraph of $G$ induced by $V \backslash S$. Note that, for all $i \in V \backslash S$,

$$
\{S \stackrel{G}{\rightsquigarrow} i\}=\{R \stackrel{H}{\rightsquigarrow} i\} .
$$

For every fixed $r \subset \Gamma$, the family $\{r \stackrel{H}{\rightsquigarrow} i\}$ for $i \in V \backslash S$ is independent of $R$ because it depends only on the orientations of the edges of $H$, while $R$ depends only on the orientations of the edges of $G$ that go from $S$ to $\Gamma$ - and these two sets of edges are disjoint. Moreover, by the induction hypothesis, the events $\{r \stackrel{H}{\rightsquigarrow} i\}$, $i \in V \backslash S$, are positively associated. Since for fixed sets $r$ and $r^{\prime}$ such that $r^{\prime} \subset r$, $\left\{r^{\prime} \rightsquigarrow i\right\} \Longrightarrow\{r \rightsquigarrow i\}$ for all vertices, we can apply Lemma 2.1 to conclude that the events $\{R \rightsquigarrow i\}, i \in V \backslash S$, are positively associated.

To conclude the proof, note that the events $\{S \rightsquigarrow i\}$ are certain for $i \in S$ and that the union of a family of positively associated events and of a family of certain events is still positively related.

\subsection{Corollaries}

Corollary 2.3. Let $G$ be a finite graph with independently oriented edges. For any target set $T$, the events $\{i \rightsquigarrow T\}, i \in V$, are positively associated.

Proof. Consider the randomly oriented graph $H$ obtained by reversing the orientation of the edges of $G$, i.e. such that $\{i \stackrel{H}{\rightarrow} j\}=\{j \stackrel{G}{\rightarrow} i\}$. Then for all $i \in V$,

$$
\{i \stackrel{G}{\rightsquigarrow} T\}=\left\{T_{\stackrel{H}{\rightsquigarrow}}^{\mapsto} i\right\},
$$


and we already know from Theorem 2.2 that the events $\{T \stackrel{H}{\rightsquigarrow} i\}, i \in V$, are positively associated.

Corollary 2.4. Let $G$ be an infinite graph with independently oriented edges. Let $f$ and $g$ be increasing, non-negative functions on $\mathbb{R}^{V}$ that depend only on a finite number of coordinates (i.e. such that there exists a finite set $U \subset V$ and $\tilde{f}: \mathbb{R}^{U} \rightarrow$ $\left[0,+\infty\left[\right.\right.$ such that $f=\tilde{f} \circ \varphi$, where $\varphi$ is the canonical surjection from $\mathbb{R}^{V}$ to $\left.\mathbb{R}^{U}\right)$. Then, for any source set $S$, letting $\mathbf{X}=\left(\mathbb{1}_{\{S \sim i\}}\right)_{i \in V}$,

$$
\mathbb{E}(f(\mathbf{X}) g(\mathbf{X})) \geqslant \mathbb{E}(f(\mathbf{X})) \mathbb{E}(g(\mathbf{X}))
$$

Proof. Let $G_{n}$ be an increasing sequence of finite graphs such that $G=\cup_{n} G_{n}$, and for all $i \in V$, let

$$
X_{i}^{(n)}=\left\{S \stackrel{G_{n}}{\rightsquigarrow} i\right\},
$$

so that $X_{i}^{(n)} \subset X_{i}^{(n+1)}$ and $X_{i}=\bigcup_{n} X_{i}^{(n)}$. Since the functions $f$ and $g$ are increasing, so are the sequences $f\left(\mathbf{X}^{(n)}\right)$ and $g\left(\mathbf{X}^{(n)}\right)$. Thus, using Theorem 2.2 and monotone convergence,

$$
\mathbb{E}\left(\lim _{n} f\left(\mathbf{X}^{(n)}\right) g\left(\mathbf{X}^{(n)}\right)\right) \geqslant \mathbb{E}\left(\lim _{n} f\left(\mathbf{X}^{(n)}\right)\right) \mathbb{E}\left(\lim _{n} g\left(\mathbf{X}^{(n)}\right)\right) .
$$

Finally, if $f$ and $g$ depend on a finite number of events $X_{i}$, then for every realization of $\mathbf{X}$ we have $\lim _{n} f\left(\mathbf{X}^{(n)}\right)=f(\mathbf{X})$ and $\lim _{n} g\left(\mathbf{X}^{(n)}\right)=g(\mathbf{X})$.

Corollary 2.5 (Narayanan, 2016). For any (possibly infinite) graph with independently oriented edges, for any source set $S$ and for any two vertices $i$ and $j$,

$$
\mathbb{P}(S \rightsquigarrow i, S \rightsquigarrow j) \geqslant \mathbb{P}(S \rightsquigarrow i) \mathbb{P}(S \rightsquigarrow j)
$$

Proof. Take $f:\left(x_{k}\right)_{k \in V} \mapsto x_{i}$ and $g:\left(x_{k}\right)_{k \in V} \mapsto x_{j}$ in Corollary 2.4.

Corollary 2.6. Let $G$ be a finite graph with independently oriented edges and vertex set $V$. For any source set $S$, let

$$
N=\sum_{i \in V \backslash S} \mathbb{1}_{\{S \rightsquigarrow i\}}
$$

denote the size of the oriented percolation cluster of $G$, and set $\lambda=\mathbb{E}(N)$. Then,

$$
d_{\mathrm{TV}}(N, \operatorname{Poisson}(\lambda)) \leqslant \min \left(1, \lambda^{-1}\right)\left(\operatorname{Var}(N)-\lambda+2 \sum_{i \in V \backslash S} \mathbb{P}(S \rightsquigarrow i)^{2}\right),
$$

where $d_{\mathrm{TV}}$ denotes the total variation distance.

Proof. This is a direct application of the Stein-Chen method to the positively related variables $\mathbb{1}_{\{S \rightsquigarrow i\}}, i \in V \backslash S$ - see e.g. Theorem 4.20 in [9].

The interest of Corollary 2.6 is that one only needs a suitable upper bound on $\operatorname{Cov}\left(\mathbb{1}_{\{S \rightsquigarrow i\}}, \mathbb{1}_{\{S \rightsquigarrow j\}}\right)$ to show that the size of the oriented percolation cluster is Poissonian. 


\section{Acknowledgments}

I thank my adviser Amaury Lambert for a careful rereading of the first draft of this manuscript, and Jean-Jil Duchamps for helpful discussions. I also thank two anonymous reviewers.

\section{References}

[1] R. Ahlswede and D. E. Daykin. An inequality for the weights of two families of sets, their unions and intersections. Probability Theory and Related Fields, 43(3):183-185, 1978.

[2] A. D. Barbour, L. Holst, and S. Janson. Poisson approximation. Oxford Studies in Probability. Oxford University Press, 1992.

[3] R. Durrett. Oriented percolation in two dimensions. The Annals of Probability, 12(4):999-1040, 1984.

[4] J. D. Esary, F. Proschan, and D. W. Walkup. Association of random variables, with applications. The Annals of Mathematical Statistics, 38(5):1466-1474, 1967.

[5] J. A. Fill and R. Pemantle. Percolation, first-passage percolation and covering times for Richardson's model on the n-cube. The Annals of Applied Probability, 3(2):593-629, 1993.

[6] C. M. Fortuin, P. W. Kasteleyn, and J. Ginibre. Correlation inequalities on some partially ordered sets. Communications in Mathematical Physics, 22(2):89-103, 1971.

[7] S. Linusson. A note on correlations in randomly oriented graphs. arXiv preprint arXiv:0905.2881, 2009.

[8] B. Narayanan. Connections in randomly oriented graphs. Combinatorics, Probability and Computing, pages 1-5, 2016.

[9] N. Ross. Fundamentals of Stein's method. Probability Surveys, 8:201-293, 2011. 